\section{Preference, Complexity, and Color Information Entropy Values for Visual Depictions of Plant and Vegetative Growth}

\author{
Rob Kuper ${ }^{1}$
}

ADDITIONAL INDEX WORDS. Shannon's information entropy, plant growth, flowering, senescence, fall foliage, winter dormancy

Summary. Few have examined the relationship between landscape color changes, landscape complexity, and laypersons' visual preference ratings. We examined whether depictions of visual changes to plant and vegetative colors affect preference ratings, estimations of complexity, and computed color information entropy values. Photographs depicted four visual states of plant growth-winter dormancy, foliation, flowering, and senescence-in color at four locations on each of three landscape architecture project sites in New York and Pennsylvania. Participants viewed and evaluated the scenes depicted in the photographs for preference $(n=52)$ and estimated the presence of complexity $(n=47)$. A multiparadigm numerical computing environment performed algorithmic functions to calculate Shannon information entropy values of perceptual and categorical colors for each photograph. The visual changes depicted significantly affected perceptual color information entropy values, but significant effects were not found in three contrasts between values for the four stages of plant and vegetative growth. Preference ratings for foliated scenes were significantly higher than those for dormant and senescent scenes. Respondents' complexity estimations for foliated scenes were lower than those of flowering and senescent, yet complexity and preference did not correlate. Preference correlated strongly and positively with perceptual color information entropy, which may help predict landscape preference. However, the presence of green foliage may affect preference more than perceptual color information entropy within scenes.

S ince emerging from the work of Berlyne (1963), Vitz (1966), and Day (1967), complexity has been repeatedly examined as a possible predictor of landscape preference. Kaplan and Kaplan (1989) included complexity in the well-known, two-by-two "preference matrix," along with coherence, mystery, and legibility. Complexity is immediately inferred and encourages exploration of the twodimensional picture plane of a scene through the presence of an abundant, diverse collection of visual characteristics, regardless of arrangement.

Department of Landscape Architecture \& Horticulture, Temple University, 580 Meetinghouse Road, Ambler, PA 19002

We are grateful to Iyad Obeid for writing the algorithm to compute perceptual color information entropy and a portion of the algorithm for obtaining categorical color pixel sums. We are indebted, as well, to Catarina Ripamonti for writing the algorithm for pixel color space conversions. Reid Overturf and the Computer Services staff at Temple University deserve thanks, too, for repeatedly providing technical support, space, and power to process digital photographs. Michael Olszewski, Sasha Eisenman, and Mary Myers reviewed an early version of the manuscript and offered clear, constructive criticism for which we are deeply appreciative. Finally, we thank two anonymous reviewers and the editorial staff for further improving this manuscript for publication.

${ }^{1}$ Corresponding author. E-mail: rkuper@temple.edu.
Numerous, distinct colors, textures, shapes, and physical dimensions of foliage, flowers, path materials, topography, and structures act as identifying attributes of complexity.

Stamps (2004) indicated that "some sort of relation between complexity and preference exist," but the magnitude and direction of correlations between preference and complexity estimations varied widely and were not replicable. He suggested that future research should relate preference ratings to the "obvious mathematical expression" of complexity, Shannon's information entropy (Shannon and Weaver, 1949; Stamps, 2002, 2003), and has computed entropy values using attributes of complexity, like color. Thus, the first purpose of this study is to objectively compute color information entropy values and investigate potential relationships with complexity estimations and preference ratings.
The second purpose of this study is to examine whether color information entropy values, preference ratings, and subjective estimations of complexity are affected by visual changes to plant and vegetative characteristics during growth. Some plant and vegetative colors in temperate climates, like the northeastern United States, evidently change throughout the year. Seasonal and diurnal meteorological changes affect the appearance of many plant flowers, foliage, seeds, and stems. Likewise, humans may alter the visual appearance of plants and vegetation at different times through various means of maintenance like mowing, controlled burns, and chemical applications ranging from fertilizers to herbicides. Therefore, plant and vegetative color changes may influence human perception and affect preference ratings and complexity estimations. In addition, changes in plant and vegetative color may affect computations of color information entropy values that are derived from a scene.

Shannon and Weaver (1949) redefined and distinguished information entropy from physical or thermodynamic entropy to measure disorder in information. Entropy values are presented in bits. Generally, as the number of bits increase, the number of alternatives, or levels of a factor in information, double. Computing entropy requires parsing information into factors, levels, and units. Following is the equation for calculating entropy:

$$
H_{\text {factor }}=-\sum_{i=1}^{n \text { levels }} P_{i} \log _{2} P_{i}
$$

where $H$ is entropy in bits, $P$ is the probability of occurrences of the level, and $i$ is the level. Entropy is zero if all the information is the same. More diverse, complex information returns higher entropy values.

Employing color as a factor in information entropy computations requires acknowledging that color may be both perceived and categorized. Perceptual colors are often classified by hue, chroma, and value and examined with the use of the

\begin{tabular}{llll}
\hline $\begin{array}{l}\text { Units } \\
\begin{array}{l}\text { To convert U.S. to SI, } \\
\text { multiply by }\end{array}\end{array}$ & U.S. unit & SI unit & $\begin{array}{l}\text { To convert SI to U.S., } \\
\text { multiply by }\end{array}$ \\
\hline 0.3048 & $\mathrm{ft}$ & $\mathrm{m}$ & 3.2808 \\
25.4 & inch(es) & $\mathrm{mm}$ & 0.0394
\end{tabular}


Munsell color system (Landa and Fairchild, 2005). In contrast, the eight chromatic categorical colorsred, orange, yellow, green, blue, purple, brown, and pink - and three achromatic colors-white, black, and gray-are not quantitatively distinguishable for standardization (Berlin and Kay, 1969). To differentiate perceptual and categorical colors simply, consider that myriad perceptual colors possessing a red hue can be created by varying chroma and value, but each could also be classified as one categorical color-red.

Identifying objective measures of perceptual and categorical colors and determining whether either affects landscape preference carries practical implications. Plant growers, scientists, designers, and installers can benefit from understanding and predicting whether people prefer landscapes containing plants and vegetation that are red, green, blue, etc., regardless of chroma or value variations (categorical colors), landscapes containing myriad variations of reds, greens, blues, etc. (perceptual colors), or neither.

Estimations of diversity - a synonym of complexity and prevalent criterion in architectural aesthetic regulations-and information entropy values have directly correlated to the (categorical) color $(r=0.97)$ of houses in designed stimuli (Stamps, 2002). Elsewhere, three studies contributed to a direct correlation between rated diversity and entropy of $r=0.87$ (Stamps, 2003). Accordingly, the following is our first hypothesis:

$\mathrm{Hl}$. Complexity estimations and color information entropy values computed from depictions of scenes will significantly and directly correlate.

Two studies suggest a positive relationship between complexity, landscape color information entropy, and preference. Respondents in one study have been positively affected by the amount and diversity of color contained within landscape simulations at establishment and maturity (Hands and Brown, 2002). In another, Jorgensen et al. (2002) surmised that different colors and combinations of flowering plants may positively affect preferences for summer depictions of an urban park having no understory, regardless of the degree of enclosure. Therefore, we present our second hypothesis:

H2. Preference ratings, complexity estimations, and color information entropy values will correlate directly and significantly.

Kuper (2013) found that experts' estimations of complexity were unaffected by the visual changes to plants and vegetation depicted in color photographs of landscape scenes categorized as early summer, late summer, and fall. Landscape architects' and other environmental professionals' preference ratings and management goals have been shown to differ or conflict with laypersons (Buhyoff et al., 1978; Daniel and Boster, 1976; Kaplan and Kaplan, 1989; Vining and Ebreo, 1991). Consequently, the effect of plant and vegetative visual changes on laypersons' complexity estimations should be examined, and estimations for broader and distinct depictions of visual changes during plant growth are needed. Kuper (2013) did not solicit estimations for dormant scenes, and the seasonal or calendrical categorization of scene depictions may have inherently included flowering plants, for instance, in two or more seasonal depictions, which could have affected responses and confounded the nonsignificant effect. Accordingly, we solicited laypersons' estimations of complexity for depictions of scenes that include winter dormant, foliated, flowering and senescent plants and vegetation and further hypothesize that:

H3. Complexity estimations for foliated scenes will not differ from those of other scenes.

The results of five studies suggest that visual changes to plant and vegetative characteristics affect preference. First, generally higher scenic beauty estimations for six areas in a national forest collected after the rainy season, when compared with before (Daniel and Boster, 1976), imply that foliated, green scenes with or without colorful flowers may receive higher preference ratings than dormant or senescent scenes. Second, Kaufman and Lohr (2004) found that computer-generated generic, mature green and red trees received positive responses from participants, "purple" and "bluish purple" trees received neutral and negative responses, and "brown" and "orange-brown" trees were disliked. Third, students in Newark, DE, rated winter as colder, more dangerous, and less exciting than spring and summer, which were both rated hotter, more exciting, conducive to play, and safer than winter (Sonnenfeld, 1969). These findings suggest that scenes depicting foliated and flowering landscapes may be perceived as positive, warmer, safer, more exciting, and more conducive to play, and therefore, receive higher preference ratings than dormant or senescent scenes. Fourth, Palmer (1990) found that scenic quality ratings gathered during the seasons depicted in photographs of hardwood forest sites in the northeastern United States resulted in nonsignificant differences between full-leaf summer and fall color scene ratings; both were significantly higher than ratings for leafless spring scenes. He suggests that the "lush and intense color" of full-leaf summer and fall color scenes make them "desirable," whereas the leafless spring condition is "colorless" and "least desirable." Responses elicited for the same scenes in mid-March resulted in a nonsignificant difference between fall color and snow-covered winter scenes. Summer scene ratings were significantly higher than fall and winter; leafless spring scene ratings were significantly lower than summer, fall, and winter. Finally, summer depictions of forest settings containing herbaceous growth and the absence of timber harvest residue were rated as more highly preferred than were the same settings as depicted in the winter (Benson and Ullrich, 1981). Thus, the presence of foliage in foliated and flowering scenes may contribute to comparatively higher preference ratings. From these findings we devised the following hypothesis for examination:

H4. Preference ratings for foliated scenes will be significantly higher than those for dormant or senescent scenes.

The results of one study imply that color information entropy values derived from depictions of landscapes may be affected by visual changes to plant and vegetative characteristics between flowering or foliation and senescence. Hendley and Hecht (1949) made "visual comparisons between natural objects" and Munsell color paper samples and found wider color ranges of objects in autumn, when compared with those in summer. Thus, we hypothesized the following:

H5. Perceptual information entropy values for foliated scenes will be 
significantly lower than those of senescent scenes.

Two studies suggest that flowering scenes are highly preferred. Respondents have shown a preference for flowering herbs and grass along an English roadside (Akbar et al., 2003) and flowers in street plantings (Todorova et al., 2004). The following final hypothesis addresses these findings:

H6. Preference ratings for foliated scenes will be significantly lower than flowering scenes.

\section{Materials and methods}

STimuli. We identified two criteria to select three sites that depict a variety of landscape settings. First, the sites had to be located in the midAtlantic region of the United States to minimize or prevent a bias for novel landscapes that might exist by eliciting responses from mid-Atlantic residents (Herzog et al., 1976; Kaplan, 1977, 1984, 1985a, 1985b). Second, each site had to have matured a minimum of 5 years to avoid confounding landscape visual changes that occur following plant and vegetative installation and establishment from those in mature landscapes, which exhibit more consistent annual visual changes. After conferring with colleagues and practicing professionals, we identified three sites that met these criteria: Avalon Park and Preserve (Stony Brook, NY), Pennswood Village (Newtown, PA), and the Storm King Art Center (SKAC, Mountainville, NY). Each site has been substantially designed and may inherently possess high degrees of preference and complexity that remain stable throughout the year.

The method for creating photographs on each site is similar to the ground-based photographic monitoring protocol by Hall (2001). During initial visits to each of these intensively managed sites, a digital camera (model DSC-P200; Sony, Tokyo, Japan) recorded color photographs of views looking ahead, behind, or away from designed pathways toward "prominent visual features or vistas (that may attract viewer attention)," which Daniel and Boster (1976) suggest is appropriate in lieu of a random walk procedure. Furthermore, we positioned the camera $\approx 6 \mathrm{ft}$ above the ground, specified a standard focal length (38 $\mathrm{mm}$ ), and pointed the camera about equally toward cardinal and intercardinal directions. To avoid potential biases introduced by the presence of people in the landscape (Herzog et al., 1976), we photographed the locations when no other visitors were present.

We used annotated site maps and hard copy reproductions of the initial photographs to locate and compose identical photographs on repeat visits. Overall, photographs portrayed four plant and vegetative visual appearanceswinter dormancy, foliation, flowering, and senescence-at each of four locations within the three sites. December, January, or early March photographs classified as winter dormant contained completely defoliated deciduous trees, brown grasses (some of which were mowed), plant litter, and no snow cover. Photographs obtained in late May depicted fully foliated deciduous trees and herbaceous plants and vegetation possessing primarily green foliage without flowers. Mid-July or midSeptember photographs represented herbaceous plants and vegetation possessing flowers. Finally, photographs obtained in early to midNovember depicted senescent plants and vegetation possessing mature seed heads, some defoliation, and fall color.

We recorded and collated 370 total photographs by site, location, and date. Of these, we selected 48 photographs ( 4 visual states $\times 3$ sites $\times$ 4 camera locations at each site) that possessed the following three attributes: a) landscape orientation; b) evident visual changes in plant and vegetative characteristics between visual states; and c) in focus and without glare.

A raster graphics editor (Photoshop version CS4; Adobe, San Jose, CA) aided formatting the photographs to dimensions of $540 \times 432$ pixels $(7.5 \times 6$ inches at 72 pixels/ inch); red, green, blue (RGB) mode (i.e., color space); and 8 bits per channel. We visually matched the composition of photographs depicting the four visual states for each location. Two methods controlled the potential effects of weather type and brightness on participants' preference ratings and complexity estimations (Beute and de Kort, 2013) due to weather and the combination of camera artifacts, direction of view, and seasonal variations in the angle of solar incidence. First, we replaced the sky in all photographs with a blue $($ red $=125$, green $=170$, blue $=220)$ to white $($ red $=255$, green $=255$, blue $=$ 255) gradient spanning the distance from the top to middle of each photograph, respectively. Second, we adjusted and equalized the brightness levels of each photograph. A public domain image-processing program (Image J version 1.47; National Institutes of Health, Bethesda, MD) calculated mean gray values of each photograph. A repeated measures analysis of variance (ANOVA) performed on mean gray values with site as a covariate using a statistical analyses software package (SPSS version 19; IBM Corp., Armonk, NY) showed that mean gray values did not significantly differ by depictions of visual change $[F(3,27)=0.368, P>$ $0.05]$ or by site $[F(2,9)=0.899, P>$ $0.05]$. Photographs for each visual change at one location for each study site are shown in Figs. 1-3.

SuRVEY InSTRUMENTS. Survey instruments contained instructions for viewing and evaluating photographs, definitions of criteria, evaluation scales, and photographs on "slides." A slide show presentation program (PowerPoint version 13; Microsoft, Redmond, WA) presented the instruments to student participants. All slides possessed a gray background $($ red $=127$, green $=127$, blue $=$ 127). The first slide of all instruments requested that participants read all instructions before beginning the experiment, presented the purpose of the study-to examine landscape preference or landscape complexityand asked participants to view and evaluate each of 56 photographs in $10 \mathrm{~s}$, which in our experience, participants have done comfortably. Two studies suggest that 5 to $8 \mathrm{~s}$ of exposure time to photographs is adequate (Brown and Daniel, 1984; Daniel and Boster, 1976). Following two slides that presented participants' rights and a confidentiality notice, the fourth slide told participants that the photographs may gradually become familiar, asked all participants to view and rate each photograph as if it was the first time they were seeing it, and to use the full range of answers provided when responding. As per 


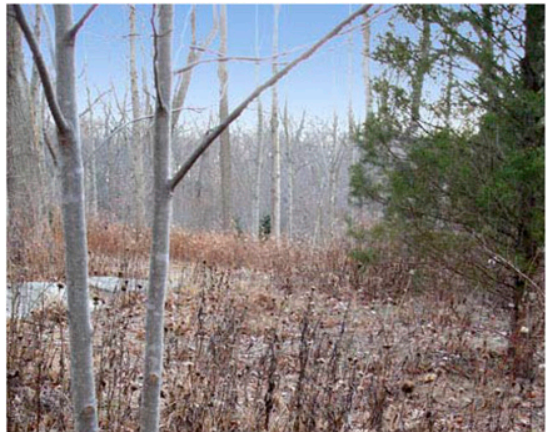

Dormant

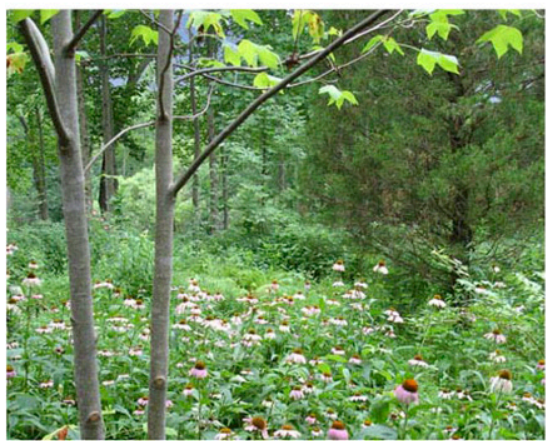

Flowering

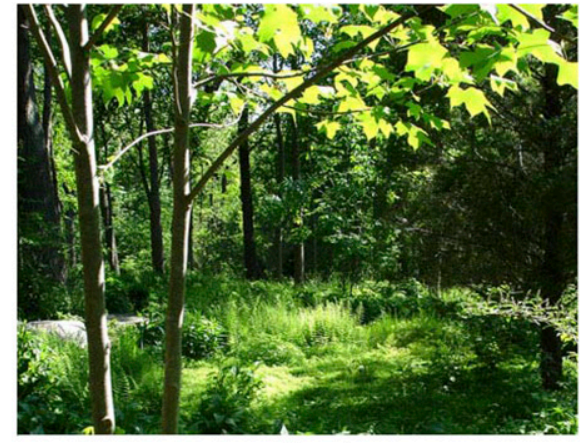

Foliated

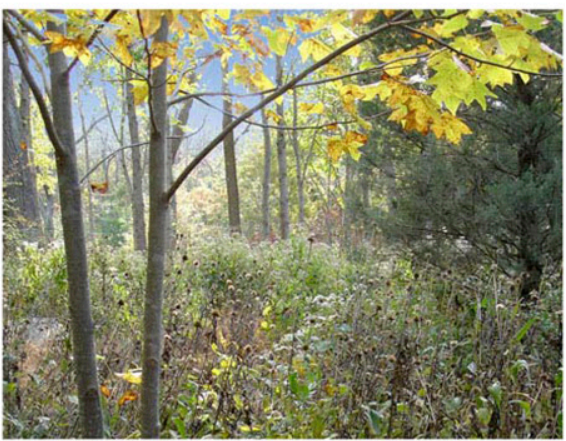

Senescent
Fig. 1. Example photographs presented to participants that depict winter dormant, foliated, flowering, and senescent plants and vegetation at Avalon Park and Preserve, Stony Brook, NY (all photographs by the author).

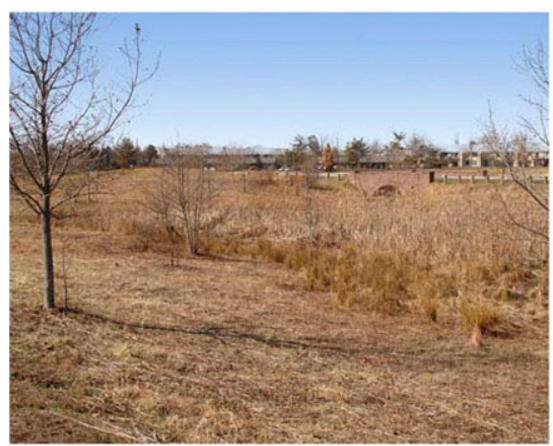

Dormant

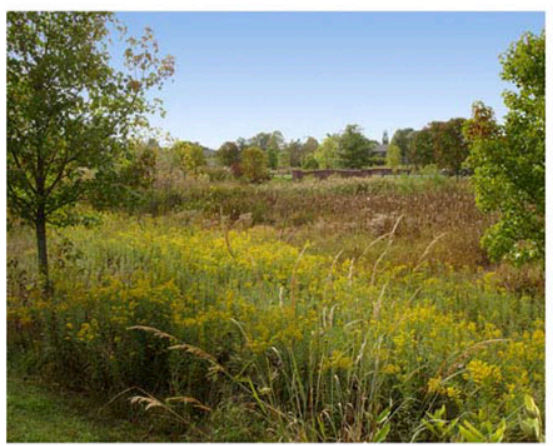

Flowering

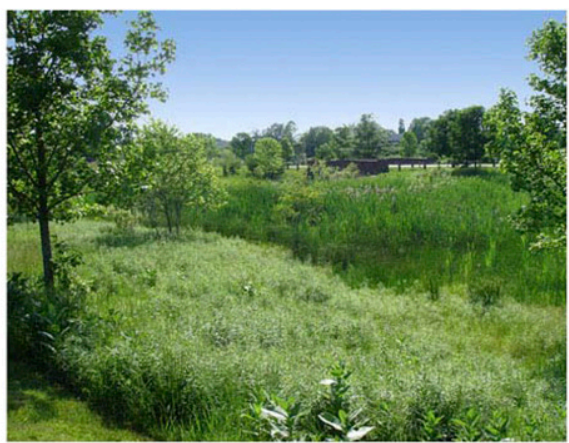

Foliated

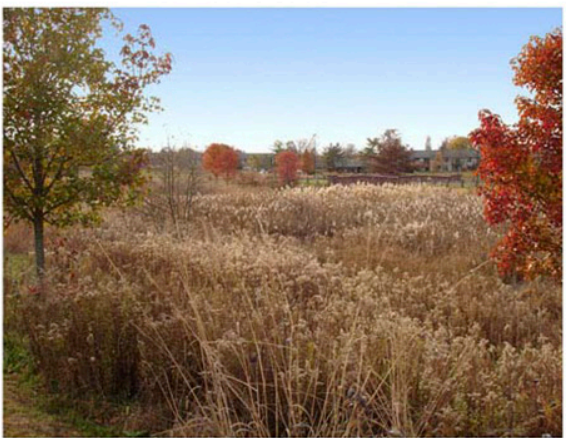

Senescent
Fig. 2. Example photographs presented to participants that depict winter dormant, foliated, flowering, and senescent plants and vegetation at Pennswood Village, Newtown, PA.
Kaplan and Kaplan (1989) and Herzog and Kropscott (2004), the fourth slide told participants providing preference ratings to "Use the following scale to rate how much you like the scene within each photograph (NOT the photo itself), for whatever reason. Please don't worry about whether you're right or wrong, or whether you agree with anyone else. What do you think?" Also like Kaplan and Kaplan (1989), the fourth slide told participants estimating the presence of complexity that "Complexity is the degree of richness or intricacy in a scene (NOT the photo)." In addition, this fourth slide asked participants to think about "How much is going on in the scene? How much is there to look at? How much does the scene contain many elements of different kinds?" Finally, the fourth slide presented a fivepoint scale that ranged from "don't like at all" (or "not complex at all"), which was equal to one point on a Likert-type scale, to "like a great deal" (or "a great deal of complexity"), which was equal to five points.

Each instrument presented all photographs on each slide at the same size and position. In addition, directly below each photograph, each instrument presented the number and location of the photograph within the overall sequence (e.g., l of 50 ). The first and last four photographs of all instruments depicted locations on the three study sites that were not represented elsewhere in the instruments. The former allowed participants to become familiar with the stimuli and rating scale; the latter attempted to control respondents' possible fatigue and any end-of-instrument effects. All participants viewed and evaluated these "filler" photographs (Herzog and Kropscott, 2004) in the same order; the data analysis excluded these responses. Each of the eight instruments randomly presented the middle 48 photographs to groups of participants in a different order; data analysis included responses for these photographs.

Procedure. The Behavioral and Social Sciences Committee of the Institutional Review Board at Temple University approved the protocol for eliciting responses from human subjects in this study. Students enrolled in general education or introductory courses within the School of Environmental Design at Temple University 


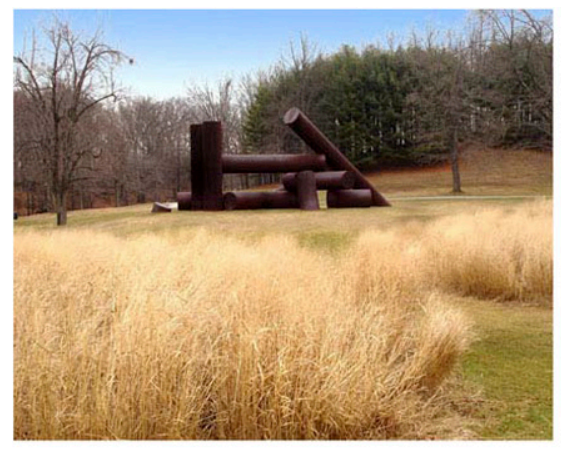

Dormant

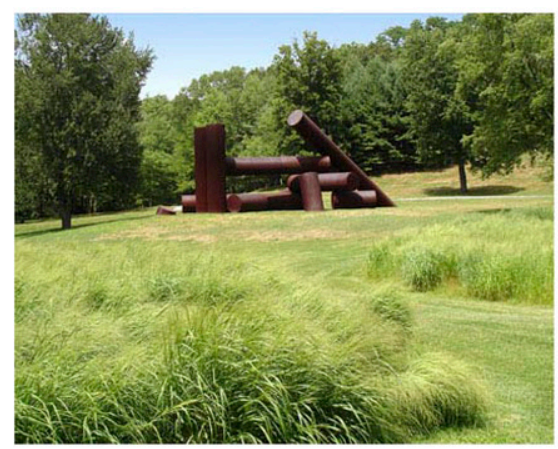

Flowering

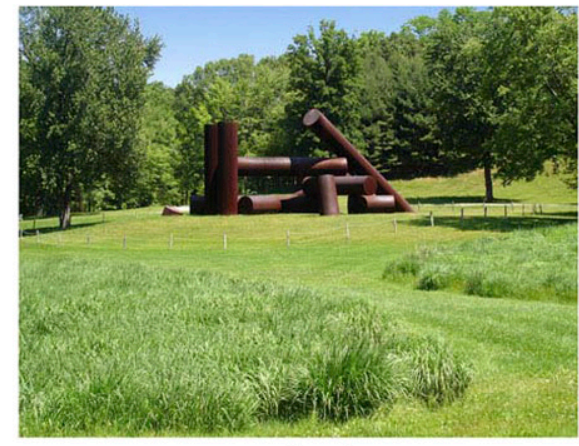

Foliated

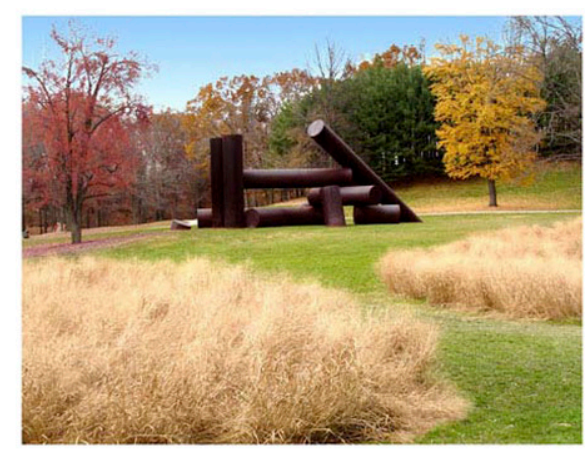

Senescent

Fig. 3. Example photographs presented to participants that depict winter dormant, foliated, flowering, and senescent plants and vegetation at The Storm King Art Center, Mountainville, NY (artwork @ The Alexander Liberman Trust).

acted as potential layperson participants. Students enrolled in introductory community planning and community development courses provided preference ratings and complexity estimations along with enrollees in "green versus gray," which the general university undergraduate population enrolls in. All students were naive as to the purpose of the study when we introduced the survey instruments, thereby controlling self-selection.

We presented the survey instruments to human subjects to elicit evaluations of the photographs. After reading survey instrument instructions, participants viewed and evaluated projections of color photographs. Participants then marked one of the number choices on a paper response sheet that corresponded to the photograph number of the slide. The top of each response sheet included evaluation scale ranges and verbiage.

Each participant completed one survey instrument and saw each photograph once. Groups of 8 to 23 participants saw one of eight total survey instruments between 29 Jan. and 24 Mar. Four instruments elicited preference ratings and four elicited complexity estimations. We removed two participants' preference responses because the students formerly majored in landscape architecture. We also removed five participants' preference ratings and six participants' complexity estimations because responses did not vary enough to indicate active participation. Thus, the dataset included 52 participants' preference ratings and 47 participants' complexity estimations.

At least three limitations exist in the materials and methods of our study. First, despite efforts to control and mediate meteorological phenomena, some photos still appeared sunnier than others. Consequently, respondents' preference ratings and estimations of complexity may have been affected. Second, photographs depicting scenes at SKAC included large sculptures that may have attracted participants' attention and affected preference ratings and complexity estimations for scenes depicted at this site. Third, a mechanical problem forced us to administer one instrument used to ascertain estimations of complexity while the classroom lights were on. Thus, estimations provided by this group may have been affected by the additional ambient light present in the classroom.

Color image computations. Computer software calculated information entropy values for each digital photograph.

An algorithm for a function created in a multiparadigm numerical computing environment (MATLAB version R2013a; MathWorks, Natick, MA) computed a color information entropy value derived from the pixels within each color image. The algorithm and details regarding its employment are available from the author by request. Analysis included the computed values returned after running the function, and are hereafter referred to as perceptual color information entropy values. The values represent the uncertainty with which pixels having unique RGB formulae (perceptual colors) occur within an image. Higher values mean that many perceptual colors are each equally present; lower values mean that a few perceptual colors occur a lot.

Another algorithm for a function created in MATLAB, colorimetrically obtained the number of categorical colors present in each photograph. The function included conversion algorithms from Westland et al. (2012) and categorical color boundary vertices from Sagawa (2000). Upon running, the function converted RGB image pixels from a three-dimensional color space to a two-dimensional space, and graphed the coordinates of each pixel. The algorithm and details regarding its employment are available from the author by request. The function then counted and displayed the numbers of pixels within nine areas on the graph that corresponded to eight chromatic categorical colors and one achromatic color (white, black, and gray were viewed singularly). We used the pixel sums acquired from the function and Eq. [1] to compute categorical color information entropy values for each digital photograph in a spreadsheet application (Excel version 2013, Microsoft). Each categorical color served as a level. Higher categorical color information entropy values mean that many categorical colors are each equally present; lower values mean that a few categorical colors occur a lot. 
Data analysis. We performed three tests in SPSS. First, all participants viewed and evaluated each of the four visual states depicted in photographs. Therefore, a repeatedmeasures ANOVA tested for possible differences in participants' meanper-scene preference ratings. Separate repeated measures ANOVA tests investigated possible differences in perceptual and categorical information entropy values. In each ANOVA test, visual state served as the independent variable and included four levelsone for each visual state of winter dormancy, foliated, flowering, and senescent.

Examinations of landscape preference and complexity have often employed stimuli that depict foliated plants and vegetation (e.g., Herzog and Kropscott, 2004; Kaplan et al., 1972; Ulrich, 1984, 1986). Ulrich (1986) cited a need for investigations of preference for landscapes containing defoliated trees, yet few, if any, have occurred. Therefore, we specified the following three simple contrasts when significant main effects resulted: values for photographs depicting foliated landscapes vs. those of winter dormancy; foliated values vs. flowering; and foliated values vs. senescent. Hereafter, "scenes" refers to the landscapes contained within the photographs.

Second, Wilcoxon signed-ranks tests performed in SPSS investigated possible differences in participants' mean-per-scene estimations of complexity. Third, Spearman rank-order correlation coefficients computed in SPSS investigated potential relationships between variables measured. Given the number of tests performed on the data, we decreased the alpha level denoting a significant effect to prevent an increase in type I errors.

\section{Results}

Color image computations. Repeated measures ANOVA results in Table 1 indicate that visual changes in plant and vegetative characteristics between scenes across three sites significantly affected perceptual color information entropy values. Cohen's (1988) $d$ suggests a moderate effect. Contrast test results showed that mean perceptual color information entropy values for foliated scenes did not significantly differ from those of winter dormancy, flowering, and senescence.

Repeated measures ANOVA results in Table 2 show that the visual changes to plant and vegetative characteristics between scenes did not significantly affect categorical color information entropy values computed for the visual states.

SURVEY INSTRUMENT RESPONSES. Repeated measures ANOVA results in Table 3 show a significant and very strong affect of visual change to plant and vegetative characteristics on respondents' preference ratings. Contrast results indicate that mean preference ratings for depictions of winter dormancy are significantly lower than those of foliation. Cohen's (1988) $d$ for this contrast suggests a very strong effect, and the probability of superiority (Grissom, 1994) implies that the foliated scene would receive a higher preference rating in $98 \%$ of randomly selected scene pairs. Preference ratings for depictions of foliation were also significantly higher than those of senescence. Cohen's (1988) $d$ here also suggests a very strong effect, and the probability of superiority (Grissom, 1994) implies that the foliated scene would receive a higher preference rating in $86 \%$ of randomly selected scene pairs. Preference ratings between depictions of foliated and flowering scenes did not significantly differ.

Wilcoxon signed-ranks test results in Table 4 show that respondents' complexity estimations for

Table 1. Repeated measures analysis of variance for perceptual color information entropy bit values computed for $\mathbf{4 8}$ digital photographs depicting scenes in four locations at each of three landscape architecture project sites in New York and Pennsylvania. Analyses included scene values.

\begin{tabular}{|c|c|c|c|c|c|c|c|c|c|c|c|c|c|}
\hline \multirow[b]{2}{*}{ Visual state $^{\mathrm{z}}$} & \multicolumn{3}{|c|}{ Site $(0-17.78 \text { scale })^{\mathrm{y}}$} & \multirow[b]{2}{*}{ Mean } & \multirow[b]{2}{*}{$S^{w}$} & \multirow[b]{2}{*}{ df } & \multirow[b]{2}{*}{$\mathbf{M S}^{\mathbf{v}}$} & \multirow[b]{2}{*}{$F$} & \multirow[b]{2}{*}{$P^{\mathbf{u}}$} & \multirow[b]{2}{*}{$\eta_{p}^{2 t}$} & \multirow[b]{2}{*}{$\eta_{G}^{2 s}$} & \multirow[b]{2}{*}{$d^{r}$} & \multirow[b]{2}{*}{$P S(\%)^{q}$} \\
\hline & $\mathbf{A P}$ & PV & SK $^{\mathrm{x}}$ & & & & & & & & & & \\
\hline Dormant & 15.74 & 14.52 & 15.10 & 15.12 & & & & & & & & & \\
\hline Flowering & 16.17 & 15.29 & 15.30 & 15.59 & & & & & & & & & \\
\hline Senescent & 16.01 & 15.05 & 15.43 & 15.49 & & & & & & & & & \\
\hline Mean & 16.05 & 14.88 & 15.24 & 15.39 & & & & & & & & & \\
\hline Visual state $\times$ subjects & & & & & 3.05 & 33 & 0.092 & & & & & & \\
\hline Foliated/dormant change & & & & & 0.754 & 1 & 0.754 & 4.47 & 0.058 & & & 0.749 & 69 \\
\hline Foliated/flowering change & & & & & 0.563 & 1 & 0.563 & 2.47 & 0.144 & & & -0.683 & 66 \\
\hline Foliated/senescent change & & & & & 0.188 & 1 & 0.188 & 0.810 & 0.387 & & & -0.284 & 58 \\
\hline
\end{tabular}

${ }^{2}$ Stimuli across four locations at each of three sites depicted four visual states of plant and vegetative growth: winter dormancy, foliation, flowering, and senescence.

y Perceptual color information entropy is limited to the number of bits possible for digital photographs containing 233,280 pixels. High values represent many perceptual colors that occur infrequently within photographs; low values represent few perceptual colors that occur frequently.

xAvalon Park and Preserve (AP), Stony Brook, NY; Pennswood Village (PV), Newtown, PA; Storm King Art Center (SK), Mountainville, NY.

wType III sum of squares.

"Mean squares.

"Significant at $P<0.007$

tPartial eta-squared, which may be used to perform a power analysis.

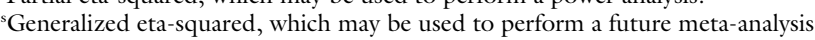

rCohen's $d$, calculated from eta-squared for the main effect, or, for contrasts, from means, sDs, and correlations.

${ }^{9}$ Probability of superiority from Grissom (1994). A 50\% means that the result is likely random; percentages increasingly closer to 100 mean that an experimental effect is increasingly likely.

$\mathrm{df}=$ degrees of freedom 
Table 2. Repeated measures analysis of variance for categorical color information entropy bit values computed for 48 digital photographs depicting scenes in four locations at each of three landscape architecture project sites in New York and Pennsylvania. Analyses included scene values.

\begin{tabular}{|c|c|c|c|c|c|c|c|c|c|c|c|c|}
\hline \multirow[b]{2}{*}{ Visual state $^{\mathrm{z}}$} & \multicolumn{3}{|c|}{ Site $(0-3.125 \text { scale })^{\mathrm{y}}$} & \multirow[b]{2}{*}{ Mean } & \multirow[b]{2}{*}{ SS $^{\mathbf{w}}$} & \multirow[b]{2}{*}{ df } & \multirow[b]{2}{*}{$\mathbf{M S}^{\mathbf{v}}$} & \multirow[b]{2}{*}{$F$} & \multirow[b]{2}{*}{$P^{\mathbf{u}}$} & \multirow[b]{2}{*}{$\eta_{p}^{2 t}$} & \multirow[b]{2}{*}{$\eta_{G}^{2 s}$} & \multirow[b]{2}{*}{$d^{r}$} \\
\hline & AP & PV & SK $^{\mathrm{x}}$ & & & & & & & & & \\
\hline Dormant & 1.54 & 2.00 & 2.08 & 1.87 & & & & & & & & \\
\hline Flowering & 1.90 & 2.17 & 1.80 & 1.96 & & & & & & & & \\
\hline Senescent & 1.90 & 2.17 & 2.27 & 2.11 & & & & & & & & \\
\hline Mean & 1.84 & 2.06 & 2.01 & 1.97 & & & & & & & & \\
\hline Visual change $\times$ subjects & & & & & 3.17 & 33 & 0.096 & & & & & \\
\hline
\end{tabular}

${ }^{z}$ Stimuli across four locations at each of three sites depicted four visual states of plant and vegetative growth: winter dormancy, foliation, flowering, and senescence.

${ }^{y}$ Categorical color information entropy values based upon the frequency of pixels that were classified as one of eight chromatic or one achromatic categorical colors within each digital photograph using a function created in MATLAB (where 0 bits $=1$ color; 1 bit $=2$ colors; 2 bits $=4$ colors; 3 bits $=8$ colors; 3.125 bits $=9$ colors). High values represent many categorical colors that occur infrequently within photographs; low values represent few categorical colors that occur frequently.

xAvalon Park and Preserve (AP), Stony Brook, NY; Pennswood Village (PV), Newtown, PA; Storm King Art Center (SK), Mountainville, NY.

wType III sum of squares.

"Mean squares.

"Significant at $P<0.007$

tPartial eta-squared, which may be used to perform a power analysis.

${ }^{s}$ Generalized eta-squared, which may be used to perform a future meta-analysis.

${ }^{\text {rCohen's }} d$, calculated from eta-squared for the main effect.

$\mathrm{df}=$ degrees of freedom

Table 3. Repeated measures analysis of variance for respondents' visual preference ratings $(n=52)$ of 48 digital photographs that depicted scenes in four locations at each of three landscape architecture project sites in New York and Pennsylvania. Analyses included mean-per-scene preference ratings.

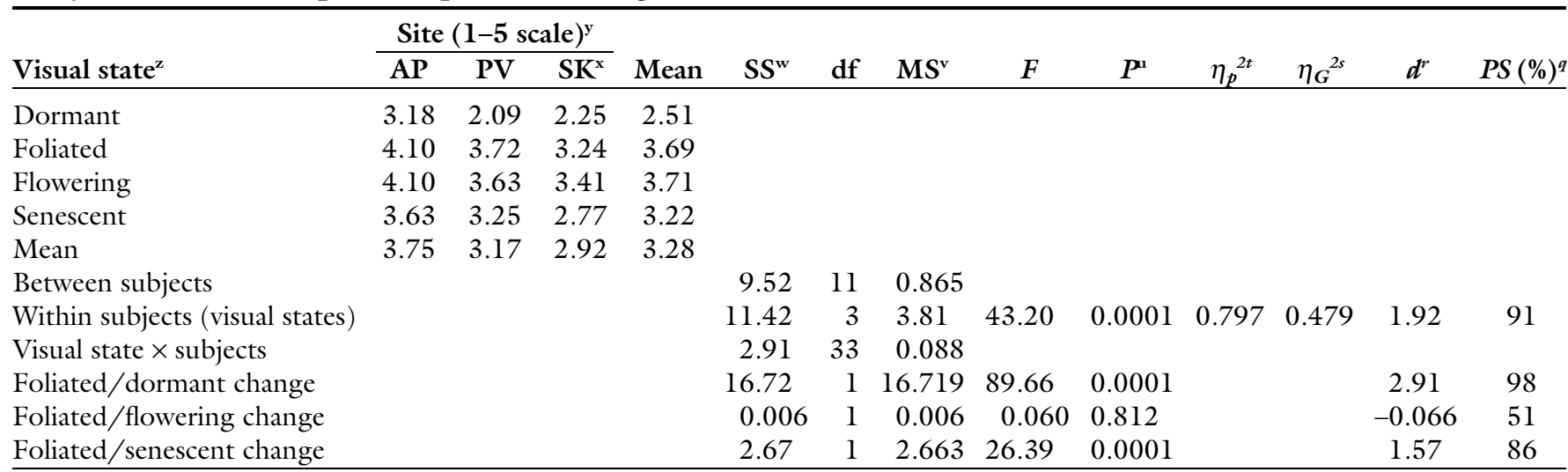

${ }^{\mathrm{z} S t i m u l i}$ across four locations at each of three sites depicted four visual states of plant and vegetative growth: winter dormancy, foliation, flowering, and senescence.

'Visual preference rating using a Likert-type scale ( $1=$ don't like at all, $5=$ like a great deal) for each landscape depiction.

xAvalon Park and Preserve (AP), Stony Brook, NY; Pennswood Village (PV), Newtown, PA; Storm King Art Center (SK), Mountainville, NY.

wType III sum of squares.

'Mean squares.

"Significant at $P<0.007$.

tPartial eta-squared, which may be used to perform a power analysis.

${ }^{s}$ Generalized eta-squared, which may be used to perform a future meta-analysis.

rCohen's $d$, calculated from eta-squared for the main effect, or, for contrasts, from means, SDs, and correlations.

qProbability of superiority from Grissom (1994). A $50 \%$ means that the result is likely random; percentages increasingly closer to 100 mean that an experimental effect is increasingly likely.

$\mathrm{df}=$ degrees of freedom

foliated scenes are significantly lower than those of flowering scenes. Also, Wilcoxon signed-ranks test results indicate that mean complexity estimations for depictions of foliated scenes are significantly lower than those of senescent scenes. In both cases, a large effect occurred.

Relationships BETWEEN VARIABLES. Table 5 shows Spearman's rho values for variables that reported a significant main effect. Perceptual color information entropy values correlated strongly to preference ratings. No other significant correlations were found.

\section{Discussion}

The significant and strong effect on preference ratings shown in Table 3 implies that participants' perceptions may have been affected by the plant and vegetative visual changes that were depicted between the photographs. In addition, the results of the contrasts suggest that participants might have liked depictions of winter dormancy and senescent plants and vegetation in the northeastern United States considerably less than those of foliated landscapes [Hypothesis 4 (H4)], yet preference ratings for depictions of flowering were not 
Table 4. Wilcoxon signed-ranks test results comparing respondents' complexity estimations $(n=47)$ for 48 digital photographs that depicted four locations at each of three landscape architecture project sites in New York and Pennsylvania. Analyses included mean-per-scene complexity estimations.

\begin{tabular}{lcccccccc}
\hline & \multicolumn{9}{c}{ Site $(\mathbf{1}-\mathbf{5} \text { scale })^{\mathrm{y}}$} & & & & & \\
\cline { 2 - 7 } Visual state $^{\mathrm{z}}$ & AP & $\mathbf{P V}$ & SK $^{\mathbf{x}}$ & Mean & Median & $Z^{\boldsymbol{v}}$ & $P^{\mathbf{v}}$ & $\tau^{\mathrm{\mu}}$ \\
\hline Dormant & 2.57 & 2.40 & 2.91 & 2.63 & 2.74 & & & \\
Foliated & 2.70 & 2.66 & 2.73 & 2.70 & 2.67 & & & \\
Flowering & 2.79 & 3.15 & 3.02 & 2.99 & 2.98 & & & \\
Senescent & 2.82 & 3.07 & 3.30 & 3.06 & 3.15 & & & \\
Foliated/dormant & & & & & & 0.746 & 0.456 & 0.108 \\
Foliated/flowering & & & & & & 2.903 & 0.004 & 0.838 \\
Foliated/senescent & & & & & & 2.825 & 0.005 & 0.816
\end{tabular}

${ }^{2}$ Stimuli across four locations at each of three sites depicted four visual states of plant and vegetative growth: winter dormancy, foliation, flowering, and senescence.

${ }^{y}$ Complexity estimations using a Likert-type scale ( $\mathrm{l}=$ not complex at all, $5=$ a great deal of complexity) for each landscape depiction.

xAvalon Park and Preserve (AP), Stony Brook, NY; Pennswood Village (PV), Newtown, PA; Storm King Art Center (SK), Mountainville, NY

"Standardized test statistic.

"Significant at $P<0.007$.

"Effect size, which is equal to $Z / \sqrt{ } \mathrm{N}$.

Table 5. Spearman rank-order correlation coefficients for an investigation of the relationships among preference, complexity, and perceptual information entropy values for $\mathbf{4 8}$ digital photographs that depicted four visual states of plant and vegetative growth in four locations on three landscape architecture project sites in New York and Pennsylvania.

\begin{tabular}{lccc}
\hline Variables $^{\mathrm{z}}$ & $\mathbf{1}$ & $\mathbf{2}$ & $\mathbf{3}$ \\
\hline 1 Perceptual information entropy & - & & \\
2 Preference & $0.560^{*}$ & - & \\
3 Complexity & 0.326 & 0.026 & - \\
\hline
\end{tabular}

${ }^{2}$ Perceptual information entropy $=$ measure of disorder in information for distinct image colors among all pixels; preference $=$ how much respondents like a scene, for whatever reason; complexity $=$ respondents' estimations of the degree of richness or intricacy in scene.

* Significant at $P<0.007$

significantly different from those of foliated scenes (H6).

One explanation why a significant and strong effect on preference ratings was found in this study is that a mathematical equivalent of complexity-color information entropy-in scenes may have affected participants' perceptions. That is, participants may have most liked scenes that each contained about equal amounts of many unique reds, greens, yellows, blues, etc., having myriad variations in lightness and richness. Finding a strong, positive correlation between perceptual color information entropy values and preference ratings, as hypothesized ( $\mathrm{H} 2)$ and presented in Table 5, suggests that perceptual color information entropy may help predict landscape preference. Table 1 shows that perceptual color information entropy values were significantly and moderately affected by visual changes to plants and vegetation. However, contrasts revealed no differences between scene means; the results do not support our hypothesis (H5). Perhaps, the overall significant visual effect plant and vegetative changes had on perceptual information entropy values reflects the visual differences that occur over the course of a year in the northeastern United States: plants and vegetation typically exhibit different, various colors to attract pollinators and reproduce, as well as during seed distribution and the cessation of chlorophyll production. Colors change gradually from $\mathrm{l} d$, week, or month to the next, but color contrasts across plant and vegetative growth states (e.g., winter dormancy and flowering) may be stark. Alternatively, scenes simulating more dramatic visual changes between visual states (e.g., foliated vs. flowering), than those depicted in our study, may result in an effect of visual change on perceptual color information entropy values between visual states. This topic may be worth pursuing in the future.

The results of this study imply that perceptual color information entropy may affect landscape preference ratings, but so too, may the predominant hue depicted in the scenes. Significantly and starkly different preference ratings between winter dormancy and foliation, then again between foliated and senescent scenes, support the finding that brown and orange-brown trees are disliked and green trees are liked (Kaufman and Lohr, 2004). Moreover, the nonsignificant difference between foliated and flowering scene preference means conflicts with suggestions that flowers made scenes more "beautiful," in at least one study (Todorova et al., 2004).

On the whole, this research related preference ratings to objectively derived color information entropy values in an effort to make landscape assessment results replicable. The absence of a relationship between preference ratings and complexity estimations herein, contrary to our hypothesis, (H2) contrasts Stamps's (2004) conclusion that "some sort of relationship exists." One explanation for this result may be that the preference ratings reflect participants' inherent associations with scenes depicting dormant and foliated vegetation. As Sonnenfeld (1969) found, participants may prefer foliated scenes because each depicts a warm, exciting, and playful environment. Complexity may have little to do with these associations, especially when participants were asked how much they liked the scenes, "for whatever reason."

Table 5 shows that a relationship between complexity and perceptual color information entropy values was not found in this study, thereby failing to support the hypothesis ( $\mathrm{Hl}$ ) derived from the results of Stamps's $(2002,2003)$ studies. One explanation may be that respondents perceived and responded to attributes of complexity in the scenes other than color, like plant and vegetative texture or height. Foliated scenes possessed immature plants and vegetation; ground surfaces were generally smooth and short. In contrast, respondents may have perceived plant and vegetative heights and textures that were more varied and pronounced, respectively, in flowering 
and senescent scenes. In addition, some defoliation in senescent scenes may have revealed vegetation and structures that were concealed in foliated scenes.

A significant relationship between preference and objectively obtained categorical color information entropy values was also not found in this study. One reason may be that few categorical colors are commonly present in predominantly natural landscapes. Table 2 shows that the mean categorical color information entropy value for each visual state is $\approx 2$ bits, which corresponds to four categorical colors. The quantity and diversity of categorical colors may have been stable across seasons, but the predominant visible colors might have changed. For instance, green predominated in foliated and flowering scenes, but brown or orange appeared most in dormant and senescent scenes. Blue skies appeared in many scenes. Higher entropy values could have resulted had other categorical colors been as visible as green and brown, which is unlikely, in most cases, given the visible appearance of plants and vegetation due to chlorophyll production and cessation, respectively, in the northeastern United States.

There are at least four reasons why complexity estimations presented in Table 4 were significantly affected by visual changes to plants and vegetative characteristics, contrary to our hypothesis (H3) and the findings in Kuper (2013). First, this study elicited responses from laypersons instead of experts, whom have been shown to have significantly different perceptions. Second, depictions in this study were classified by four distinct visual changes, regardless of the time of year. In Kuper (2013), the photographs presented to participants were classified chronologically by three seasons rather than by plant and vegetative changes associated with plant growth. Third, participants in this study provided responses to only one criterion. Participants in Kuper (2013) may have become fatigued or confounded by having to evaluate the scenes for two criteria. Finally, meteorological phenomena were controlled in this study but not in Kuper (2013): experts' perceptions may have been affected by cloud cover and varying degrees of lightness, as Beute and de Kort (2013) found.

At least three avenues of future work stem from the results of this study. First, researchers could investigate whether visual changes to plants and vegetation in climatic regimes that visually differ from the northeastern United States affect preference ratings, complexity estimations, and perceptual color information entropy values. Second, researchers could undertake an examination of whether visual changes in plant and vegetative characteristics affect estimations of other criteria used to predict preference-mystery, coherence, and particularly legibility, which was found to be significantly affected by the season depicted in photographs (Kuper, 2013). Furthermore, identifying and testing objective measures of these criteria are needed to make results replicable. Third, additional objective measures of complexity should be identified. Machine-generated image segmentation may be a viable method of objectively parsing images into various levels of texture so that textural image entropy can be computed, analyzed, and correlated to preference ratings and complexity estimations. Comparing these with human-segmented images may also reveal interesting findings.

The results of this study may generally guide practitioners in creating preferred landscapes throughout the year in the northeastern United States, and possibly, climates wherein plants and vegetation exhibit similar visual changes in response to seasonal and diurnal variations. Environmental designers and managers; plant scientists, growers, and installers should generally consider the colors that plants and vegetation display during foliation, flowering, senescence, and dormancy. Installing evergreen trees and shrubs will ensure the presence of green when most deciduous and herbaceous plants are dormant, defoliated, and brown in winter; preference may, consequently, hold steady rather than drop during winter dormancy and senescence. Plants that produce colorful berries or stems may further bolster preference and perceptual color information entropy, though likely not to the same degree as evergreen plants. Plants that emerge from winter dormancy early and enter winter dormancy late relative to other plants could, additionally, prolong high landscape preference ratings.

\section{Literature cited}

Akbar, K.F., W.H.G. Hale, and A.D. Headley. 2003. Assessment of scenic beauty of the roadside vegetation in northern England. Landsc. Urban Plan. 63:139-144.

Benson, R.E. and J.R. Ullrich. 1981. Visual impacts of forest management activities: Findings on public preferences. U.S. Dept. Agr., Intermountain Forest Range Expt. Sta., Res. Paper INT-262.

Berlin, B. and P. Kay. 1969. Basic color terms. Univ. California Press, Berkeley, CA.

Berlyne, D.E. 1963. Complexity and incongruity variables as determinants of exploratory choice and evaluative ratings. Can. J. Psychol. 17:274-290.

Beute, F. and Y.A.W. de Kort. 2013. Let the sun shine: Measuring explicit and implicit preference for environments differing in naturalness, weather type, and brightness. J. Environ. Psychol. 36:162-178.

Brown, T.C. and T.C. Daniel. 1984. Modeling forest scenic beauty. Concepts and application to ponderosa pine. U.S. Dept. Agr., Rocky Mountain Forest Range Expt. Sta., U.S. Forest Res. Paper RM-256.

Buhyoff, G.J., J.D. Wellman, H. Harvey, and R.A. Fraser. 1978. Landscape architects' interpretations of people's landscape preferences. J. Environ. Mgt. 6:255-262.

Cohen, J. 1988. Statistical power analysis for the behavioral sciences. Erlbaum, Hillsdale, NJ.

Daniel, T.C. and R.S. Boster. 1976. Measuring landscape esthetics: The scenic beauty estimation method. U.S. Dept. Agr., Rocky Mountain Forest Range Expt. Sta., U.S. Forest Res. Paper RM-167.

Day, H. 1967. Evaluation of subjective complexity, pleasingness, and interestingness for a series of random polygons varying in complexity. Percept. Psychophys. 2:281-286.

Grissom, R.J. 1994. Probability of the superior outcome of one treatment over another. J. Appl. Psychol. 79:314-316.

Hall, F.C. 2001. Ground-based photographic monitoring. U.S. Dept. Agr., Pacific Northwest Res. Sta., U.S. Forest Res. Gen. Tech. Rpt. PNW-GTR-503.

Hands, D.E. and R.D. Brown. 2002. Enhancing visual preference of ecological rehabilitation sites. Landsc. Urban Plan. 58:57-70. 
Hendley, C.D. and S. Hecht. 1949. The colors of natural objects and terrains, and their relation to visual color deficiency. J. Opt. Soc. Amer. 39:870-873.

Herzog, T.R., R. Kaplan, and S. Kaplan. 1976. The prediction of preference for familiar urban places. Environ. Behav. $8: 627-645$.

Herzog, T.R. and L.S. Kropscott. 2004. Legibility, mystery, and visual access as predictors of preference and perceived danger in forest settings without pathways. Environ. Behav. 36:659-677.

Jorgensen, A., J. Hitchmough, and T. Calvert. 2002. Woodland spaces and edges: Their impact on perception of safety and preference. Landsc. Urban Plan. 60:135-150.

Kaplan, R. 1977. Preference and everyday nature: Method and application, p. 235250. In: D. Stokols (ed.). Perspectives on environment and behavior: Theory, research and applications. Plenum, New York, NY.

Kaplan, R. 1984. Wilderness perception and psychological benefits: An analysis of a continuing program. Leis. Sci. 6:271-290.

Kaplan, R. 1985a. The analysis of perception via preference: A strategy for studying how the environment is experienced. Landscape Planning 12:161-176.

Kaplan, R. 1985b. Nature at the doorstep: Residential satisfaction and the nearby environment. J. Archit. Plann. Res. 2:115-127.
Kaplan, R. and S. Kaplan. 1989. The experience of nature. Cambridge Univ. Press, Cambridge, UK.

Kaplan, S., R. Kaplan, and J.S. Wendt. 1972. Rated preference and complexity for natural and urban visual material. Percept. Psychophys. 12:354-356.

Kaufman, A.J. and V.I. Lohr. 2004. Does plant color affect emotional and physiological responses to landscapes? Acta Hort. 639:229-233.

Kuper, R. 2013. Here and gone: The visual effects of seasonal changes in plant and vegetative characteristics on landscape preference criteria. Landscape J. 32:65-78.

Landa, E.R. and M.D. Fairchild. 2005. Charting color from the eye of the beholder. Am. Sci. 93:436-443.

Palmer, J.F. 1990. Aesthetics of the northeastern forest: The influence of season and time since harvest, p. 185-190. In: T. More, M.P. Donnelly, D.A. Graefe, and J.J. Vaske (eds.). Proc. 1990 Northeastern Recreation Researchers Symp. U.S. Dept. Agr., Northeastern Forest Expt. Sta., Gen. Tech. Rpt. NE-145.

Sagawa, K. 2000. Visual comfort evaluated by number of categorical colors in a colored image. Color Res. Appl. 25:193-199.

Shannon, C.E. and W. Weaver. 1949. The mathematical theory of communication. Univ. Illinois Press, Urbana, IL.
Sonnenfeld, J. 1969. Equivalence and distortion of the perceptual environment. Environ. Behav. 1:83-99.

Stamps, A.E., III. 2002. Entropy, visual diversity, and preference. J. Gen. Psychol. 129:300-320.

Stamps, A.E., III. 2003. Advances in visual diversity and entropy. Environ. Plann. B Plann. Des. 30:449-463.

Stamps, A.E., III. 2004. Mystery, complexity, legibility, and coherence: A metaanalysis. J. Environ. Psychol. 24:1-16.

Todorova, A., S. Asakawa, and T. Aikoh. 2004. Preference for attitudes towards street flowers and trees in Sapporo, Japan. Landsc. Urban Plan. 69:403-416.

Ulrich, R.S. 1984. View through a window may influence recovery from surgery. Science 224:420-421.

Ulrich, R.S. 1986. Human responses to vegetation and landscapes. Landsc. Urban Plan. 13:29-44.

Vining, J. and A. Ebreo. 1991. Are you thinking what I think you are? A study of actual and estimated goal priorities and decision preferences of managers, environmentalists, and the public. Soc. Nat. Resources 4:177-196.

Vitz, P.C. 1966. Preference for different amounts of visual complexity. Behav. Sci. 11:105-114.

Westland, S., C. Ripamonti, and V. Cheung. 2012. Computational color science using MATLAB. Wiley, Chichester, UK. 